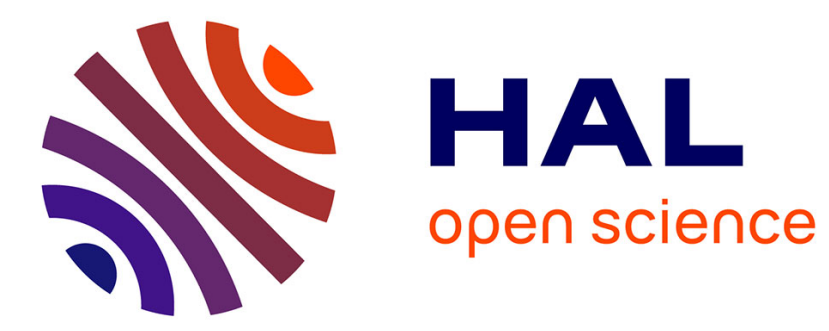

\title{
Using Maple to analyse parallel robots
}

Damien Chablat, Guillaume Moroz, Fabrice Rouillier, Philippe Wenger

\section{To cite this version:}

Damien Chablat, Guillaume Moroz, Fabrice Rouillier, Philippe Wenger. Using Maple to analyse parallel robots. Jürgen Gerhard; Ilias Kotsireas. Maple in Mathematics Education and Research, Springer, Cham, pp.50-64, 2020, Maple in Mathematics Education and Research, 978-3-030-41257-9. 10.1007/978-3-030-41258-6_4 . hal-02289699

\section{HAL Id: hal-02289699 \\ https://hal.science/hal-02289699}

Submitted on 18 Sep 2019

HAL is a multi-disciplinary open access archive for the deposit and dissemination of scientific research documents, whether they are published or not. The documents may come from teaching and research institutions in France or abroad, or from public or private research centers.
L'archive ouverte pluridisciplinaire HAL, est destinée au dépôt et à la diffusion de documents scientifiques de niveau recherche, publiés ou non, émanant des établissements d'enseignement et de recherche français ou étrangers, des laboratoires publics ou privés. 


\title{
Using Maple to analyse parallel robots.
}

\author{
Damien Chablat ${ }^{1}$, Guillaume Moroz ${ }^{2}$, Fabrice Rouillier ${ }^{3}$, and Philippe Wenger ${ }^{1}$ \\ 1 Laboratoire des Sciences du Numérique de Nantes, UMR CNRS 6004, Nantes, \\ France. \\ 2 INRIA Nancy-Grand Est \& LORIA, Nancy, France. \\ 3 INRIA Paris \& IMJ-PRG, Paris, France
}

\begin{abstract}
We present the SIROPA Maple Library which has been designed to study serial and parallel manipulators at the conception level. We show how modern algorithms in Computer Algebra can be used to study the workspace, the joint space but also the existence of some physical capabilities w.r.t. to some design parameters left as degree of freedom for the designer of the robot.
\end{abstract}

\section{Introduction}

Compared to classical numerical computations, algebraic computations make possible to work with formal parameters and equalities. In particular, this allows to study singularities exactly. One major drawback when using exact strategies is the cost of the computations which often limitate the range of reachable problems.

In the present contribution, we present the SIROPA Maple Library which has been designed to study serial and parallel manipulators at the conception level. We show how modern algorithms in Computer Algebra can be used to study the workspace, the joint space but also the existence of some physical capabilities w.r.t. to some design parameters left as degree of freedom for the designer of the robot.

For illustrating the article, we choose to study 3-PPPS mechanisms because these are 6-degree-of-freedom parallel robots but where projections with respect to 3 of the coordinates can be combined to get the full information (thus we can have explicit plots to give the right intuition).

\section{Manipulators and kinematics problems}

A serial robot (see [9]) consists in a number of rigid links connected with joints. In general the joints are active revolute or prismatic while the links are passive, parallel or intersecting.

A parallel robot (see [12]) consists in several simple serial chains that support a single platform. A well know parallel robot is the Gough-Stewart platform where the serial chains are made of 6 active actuators connected to the ground and to the platform using passive spherical joints. 

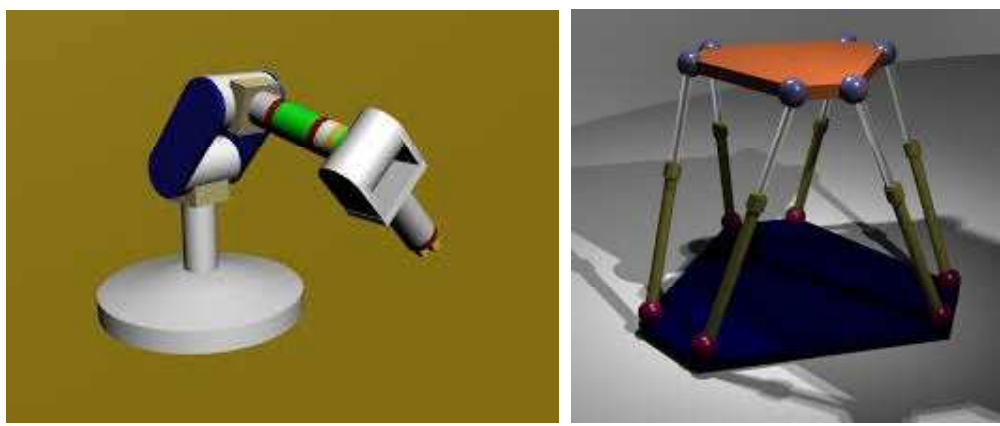

Fig. 1. Serial (left) and Parallel (right) manipulators

The (active) joint values (positions of the prismatic joints, angles of the revolute joints) and the end effector positiond are linked by the so called kinematic equations which can be turned into an equivalent system of algebraic equations. The solution set of the kinematic equations will be called the kinematic variety in this paper.

The direct kinematic problem consists in computing the possible positions of the end effector of the robot knowing the joint values.

The inverse kinematic problem consists in computing the possible joint values knowing the position of the end-effector. It is used, for example, to compute the theoretical instructions to give to the actuators in order to follow a given trajectory.

These problems might have several solutions. For example, for a generic Gough-Stewart platform, the direct kinematics problem might have up to 40 solutions [7].

A posture is associated with one solution to the inverse geometric problem of a robot while a position is associated with one solution to the direct kinematics problem. The set of points that can be reached by the end-effector is named the workspace.

Mathematically, studying the direct or the inverse kinematics problem remains to study the same system of kinematics equations but with unknowns playing different roles.

For the direct kinematics problem, the joint's unknowns play the role of parameters while the positions's unknowns play the role of variables : we study the structure (existence, number, multiplicity, etc.) of the positions w.r.t. the joint's values. For the inverse kinematics problem, the positions' unknowns play the role of parameters while the joint's unknowns play the role of variables: we study the structure (existence, number, multiplicity, etc.) of the joints w.r.t. the positions values.

In both cases, one has to consider an agebraic variety living in some ambiant space of dimension $n=d+k$ with a variable's subspace of dimension $d$ and a paremeters' subspace of dimension $k$. The goal is then to decompose the pa- 
rameter's space into regions above which the variables describe regular regions where, in short, the implicit theorem could apply, and regions where something bad happens (solutions collaps, go to infinity, etc.). This means that, above the favorable regions, the direct (or the inverse) kinematics problem has a finite and constant number of solutions and each solution can easily be followed when the parameters vary.

\section{The SIROPA Maple Library}

SIROPA is a MAPLE library (see [8] for an eshaustive description) developed to analyze the singularities, workspace and joint space of serial and parallel manipulators as well as tensegrity structures [14]. There are two main parts of the library shown in Figure 2, the first one provides the algebraic tools to solve the kinematic equations, including conversions of trigonometric equations in the algebraic form. The other one, SIROPA, provides modeling, analyzing and plotting functions for different manipulators, shown in Figure 3.

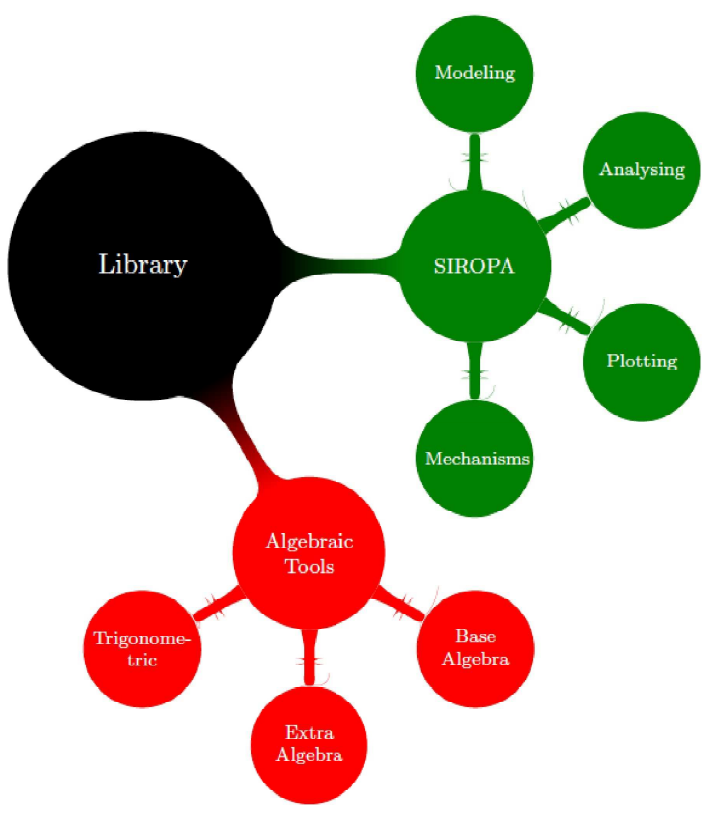

Fig. 2. Architecture of Library 


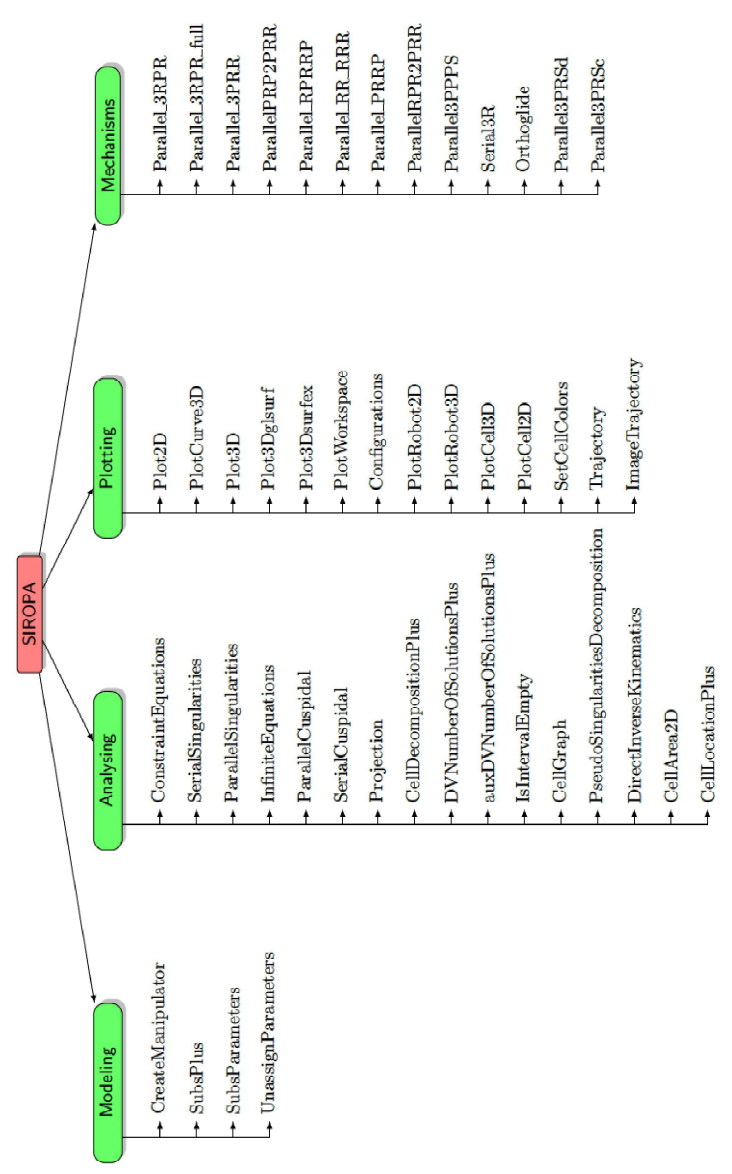

Fig. 3. List of all the defined functions in SIROPA library

The main algebraic tools we need for our work are related to the study of parametric systems of polynomial equations with rational (or floating point) coefficients with generically finitely many complex solutions.

One key point is to be able to provide a partition of the parameter's space in regions/subsets over which a given parametric system has a constant number of real solutions and tools to characterize each region : computing the constant number of solutions over a given region, drawing the region when possible, deciding if two points are in the same region, etc.

Such a partition is naturally given by the so called (minimal) Discriminant Variety [10] associated with the projection onto the parameter's space.

Let us denote by $S=\left\{f_{1}(T, X)=0, \ldots, f_{l}(T, X)=0\right\}$ the system we want to study, where $T=T_{1}, \ldots, T_{d}$ is the set of parameters, $X=X_{1}, \ldots, X_{n}$ is the 
set of unknowns, the $f_{i}, i=1 \ldots l$ being polynomials in the indeterminates $T, X$, with rational coefficients.

We suppose that $S=\left\{f_{1}(T, X)=0, \ldots, f_{l}(T, X)=0\right\}$ has generically finitely many complex solutions, which is the case for our studies, that is to say, for almost all the d-uples $\left(t_{1}, \ldots, t_{d}\right) \in \mathbb{C}^{d}$, the system $S_{\mid T=t}=\left\{f_{1}(t, X)=\right.$ $\left.0, \ldots, f_{l}(t, X)=0\right\}$ has finitely many complex solutions. The complex solutions of $S$ thus define an algebraic variety denoted by $V(I)$, with $I$ being the ideal of $\mathbb{Q}[T, X]$ generated by the polynomials $f_{1}, \ldots, f_{l}$, such that $\overline{\Pi_{T}(V(I))}=\mathbb{C}^{d}$, where $\Pi_{T}$ denotes the projection onto the parameter's space and where $\bar{W}$ denotes the closure of any subset $W \subset C^{d}$

Let's consider the definition (adapted from [10]) of The (minimal) Discriminant Variety of $V(I)$ wrt $\Pi_{T}$, denoted $W_{D}$ in the sequel, is the smallest algebraic variety of $\mathbb{C}^{d}$ such that given any simply connected subset $\mathcal{U}$ of $\mathbb{R}^{d} \backslash W_{D}$, the number of real solutions of $\mathcal{S}$ is constant over $\mathcal{U}$.

In our context, the minimal Discriminant Variety of $V(I)$ wrt $\Pi_{T}$, will always be the union of

- $W_{\mathrm{sd}}$ : the closure of the projection by $\Pi_{T}$ of the components of $V(I)$ of dimension $<d$

- $W_{c}$ : the union of the closure of the critical values of $\Pi_{T}$ in restriction to $V(I)$ and of the projection of the singular values of $V(I)$

- $W_{\infty}$ : the set of $u=\left(u_{1}, \ldots, u_{d}\right)$ such that $\Pi_{T}^{-1}(\mathcal{U}) \cap V(I)$ is not compact for any compact neightborhood $\mathcal{U}$ of $u$ in $\Pi_{T}(V(I))$.

An important remark is that if $l=n$ (say if the system has as many polynomials as unknowns), then $W_{\mathrm{sd}}=\emptyset$.

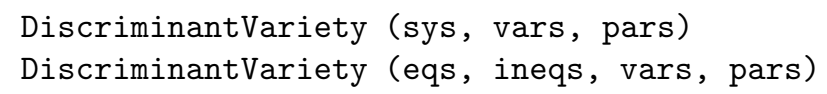

sys list of equations and strict inequalities between polynomials with rational coefficient

vars list of names; the indeterminates

pars (optional) list of names; the parameters

eqs list of polynomials $f$ with rational coefficients representing equations of the form $f=0$

ineqs list of polynomials $g$ with rational coefficients representing constraint inequalities of the form $0<g$

The function DiscriminantVariety(eqs, ineqs, vars, pars) available through the SIROPA Package computes a discriminant variety of the system

$$
[f=0,0<g]_{f \in \text { eqs }}, \quad g \in \text { ineqs }
$$

of equations and inequalities with respect to the indeterminates vars and the parameters pars. 
The input system must satisfy the following properties:

- There are at least as many equations as indeterminates.

- At least one and at most finitely many complex solutions exist for almost all complex parameter values (the system is generically solvable and generically zero-dimensional).

- For almost all complex parameter values, there are no solutions of multiplicity greater than one (the system is generically radical). In particular, the input equations are square-free.

An error occurs if one of these three previous conditions is violated.

- The result is returned as a list of lists of polynomials in pars such that the discriminant variety is the union of the set of solutions of the polynomials in each inner list.

- If pars is not specified, it defaults to all the names in sys that are not indeterminates.

- This function attempts to find a minimal discriminant variety, but it may return a proper superset in the case that it does not succeed.

- The discriminant variety is computed using Gröbner basis techniques and thus, the choice of ordering of the variables might be critical for efficiency, some intermediate objects could be huge.

A cylindrical algebraic decomposition (CAD) of the n-dimensional real space is a partition of the whole space into connected semi-algebraic subsets such that the cells in the partition are cylindrically arranged, that is, the projection of any two cells onto any lower dimensional real space is either equal or disjoint. This decomposition is called F-invariant if, for any given cell, the sign of each polynomial in $\mathrm{F}$ does not change over the cell. CylindricalAlgebraicDecompose $(\mathrm{F}, \mathrm{R})$ returns an $\mathrm{F}$-invariant $\mathrm{CAD}$ of the $n$-dimensional real space, where $n$ is the number of variables in $\mathrm{R}$. This assumes that $\mathrm{R}$ has characteristic zero and no parameters, such that the base field of $\mathrm{R}$ is the field of rational numbers [1].

In our case, we make use of a partial CAD sometimes named Open CAD to decompose the parameter space of a parametric polynomial system into the union of the discriminant variety and a collection of cells in which the original system has a constant number of solutions (see [6] and [11] for similar contexts). The open CAD is thus the union of the discriminant variety and of the cells of maximum dimension of the $\mathrm{CAD}$ associated to the discriminant variety.

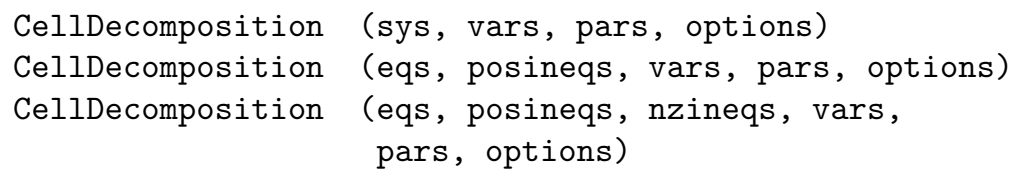




\begin{tabular}{|l|l|}
\hline sys & $\begin{array}{l}\text { list of equations and strict inequalities between } \\
\text { polynomials with rational coefficients }\end{array}$ \\
\hline vars & list of names; the indeterminates \\
\hline pars & (optional) list of names; the parameters \\
\hline eqs & $\begin{array}{l}\text { list of polynomials } f \text { with rational coefficients } \\
\text { representing equations of the form } f=0\end{array}$ \\
\hline posineqs & $\begin{array}{l}\text { list of polynomials } g \text { with rational coefficients } \\
\text { representing constraint inequalities of the form } 0<g\end{array}$ \\
\hline nzineqs & $\begin{array}{l}\text { list of polynomials } g \text { with rational coefficients } \\
\text { representing constraint inequations of the form } g \neq 0\end{array}$ \\
\hline options & $\begin{array}{l}\text { sequence of optional equations of the form } \\
\text { keyword=value where keyword is either } \\
\text { output or method }\end{array}$ \\
\hline
\end{tabular}

The function returns a data structure that can be used for (examples):

- Plotting the regions of the parameter space for which the system has a given number of solutions.

- Extracting sample points in the parameter space for which the system has a given number of solutions.

- Extracting boxes in the parameter space in which the system has a given number of solutions.

The record returned captures information about the solutions of the system depending on the parameter values, including:

- a discriminant variety;

- for each full-dimensional open cell, a sample point strictly in the interior of the cell; if possible, the coordinates of the sample point are chosen to be integers.

The input system must satisfy the same properties as for the discrimant variety.

\section{Case Studies on some 3-PPPS manipulators}

For illustrating this contribution, we will study some 3-PPPS manipulators : each leg is composed of three orthogonal prismatic joints $(\mathrm{P})$ and one spherical joint $(\mathrm{S})$, the first two prismatic joints being actuated. These 6-degree-of-freedom robots have the particularity to have independent sets of freedom motions i.e. 3 translations and 3 rotations and thus allows us to have 3 dimensional plots illustrating various kinds of singularities.

\subsection{Joint space and workspace analysis}

For illustrating the way the SIROPA Library can be used for studying the workspace of a given manipulator, we chose a 3-PPPS parallel robot derived 
from [4] with an equilateral mobile platform and a U-shape base and use quaternion parameters to represent the aspects [3], i.e. the singularity free regions of the workspace. The three legs are identical and made with two actuated prismatic joints plus one passive prismatic joint and a spherical joint. The axes of first three joints form an orthogonal reference frame.

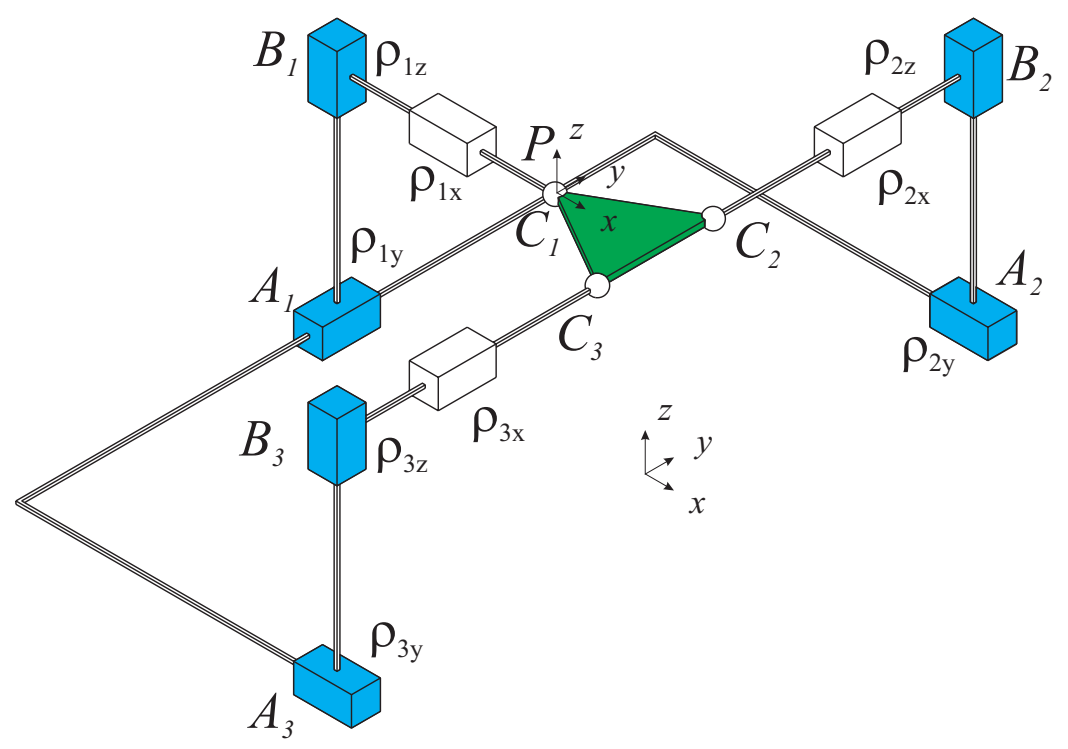

Fig. 4. The 3-PPPS parallel robot and its parameters in its "home" pose with the actuated prismatic joints in blue, the passive joints in white and the mobile platform in green

The coordinates of the moving platform in the fixed reference frame can be expressed using a general rotation matrix as $\boldsymbol{W}_{i}=\mathbf{R} \mathbf{V}_{i}+\boldsymbol{P}$, where $\left(\boldsymbol{V}_{i}\right)_{i=1 \ldots 3}$ are the 3 points describing the moving frame, $\boldsymbol{P}=[x, y, z]^{T}$ is a translation vector, and $\boldsymbol{R}=\left[\begin{array}{lll}u_{x} & v_{x} & w_{x} \\ u_{y} & v_{y} & w_{y} \\ u_{z} & v_{z} & w_{z}\end{array}\right]$ a rotation which can be parametrized by unit quaternions as follows:

$$
\boldsymbol{R}=\left[\begin{array}{ccc}
2 q_{1}^{2}+2 q_{2}^{2}-1 & -2 q_{1} q_{4}+2 q_{2} q_{3} & 2 q_{1} q_{3}+2 q_{2} q_{4} \\
2 q_{1} q_{4}+2 q_{2} q_{3} & 2 q_{1}^{2}+2 q_{3}^{2}-1 & -2 q_{1} q_{2}+2 q_{3} q_{4} \\
-2 q_{1} q_{3}+2 q_{2} q_{4} & 2 q_{1} q_{2}+2 q_{3} q_{4} & 2 q_{1}^{2}+2 q_{4}^{2}-1
\end{array}\right]
$$

with $q_{1} \geq 0$. Choosing for $\boldsymbol{V}_{1}$ and $\boldsymbol{V}_{2}$ one corner and the median of the triangle modelling the moving platform, ( $\boldsymbol{V}_{3}$ is then fixed by the two other points), say $\boldsymbol{V}_{1}=[0,0,0]^{T}, \boldsymbol{V}_{2}=[\sqrt{3} / 2,1 / 2,0]^{T}$ and $\boldsymbol{V}_{3}=[\sqrt{3} / 2,-1 / 2,0]^{T}$ appeared to be the best choice. 
Obviously, one then has to consider additional constraints equations in order to describe the links and the geometry of the moving platform. The points $A_{i}$ and $C_{i}$ can be expressed w.r.t. to the joints variables

$$
\begin{aligned}
& \boldsymbol{A}_{1}=\left[2, \rho_{1 y}, \rho_{1 z}\right]^{T} \quad \boldsymbol{C}_{1}=\left[\rho_{1 x}, \rho_{1 y}, \rho_{1 z}\right]^{T} \\
& \boldsymbol{A}_{2}=\left[-\rho_{2 y}, 2, \rho_{2 z}\right]^{T} \quad \boldsymbol{C}_{2}=\left[-\rho_{2 y}, \rho_{2 x}, \rho_{2 z}\right]^{T} \\
& \boldsymbol{A}_{3}=\left[\rho_{3 y},-2, \rho_{3 z}\right]^{T} \quad \boldsymbol{C}_{3}=\left[\rho_{3 y},-\rho_{3 x}, \rho_{3 z}\right]^{T}
\end{aligned}
$$

and the fact that there are 3 passive joints $\left(\rho_{1, x}, \rho_{2, x}, \rho_{3, x}\right)$ leads also to additional simple algebraic rules : $\rho_{1 x}=x, \rho_{2 x}=u_{y} \sqrt{3} / 2+v_{y} / 2+y$ and $\rho_{3 x}=$ $-u_{y} \sqrt{3} / 2+v_{y} / 2-y$. We finally obtain the following kinematic equations which are algebraic when adding the formal equation $\left(\sqrt{3}^{2}-3=0\right.$, considering $\sqrt{3}$ as a symbol:

$$
\begin{array}{lrl}
\rho_{1 y}-y & =0 \\
\rho_{1 z}-z & =0 \\
\left(-2 q_{1}^{2}-2 q_{2}^{2}+1\right) \sqrt{3} / 2+q_{1} q_{4}-q_{2} q_{3}-x-\rho_{2 y} & =0 \\
\sqrt{3}\left(q_{1} q_{3}-q_{2} q_{4}\right)-q_{1} q_{2}-q_{3} q_{4}+\rho_{2 z}-z & =0 \\
\left(-2 q_{1}^{2}-2 q_{2}^{2}+1\right) \sqrt{3} / 2-q_{1} q_{4}+q_{2} q_{3}-x+\rho_{3 y} & =0 \\
\sqrt{3}\left(q_{1} q_{3}-q_{2} q_{4}\right)+q_{1} q_{2}+q_{3} q_{4}+\rho_{3 z}-z & =0
\end{array}
$$

\section{Joint space analysis}

Analyzing the joint space consists in computing a partition of this space in regions above which the DKP has a fixed number of solutions. In fact, as we are not interested in regions of dimension less than 3 , so that we compute the discriminant variety of the kinematics variety w.r.t. the projection onto the joint space and then describe the complement of this variety using an open-CAD.

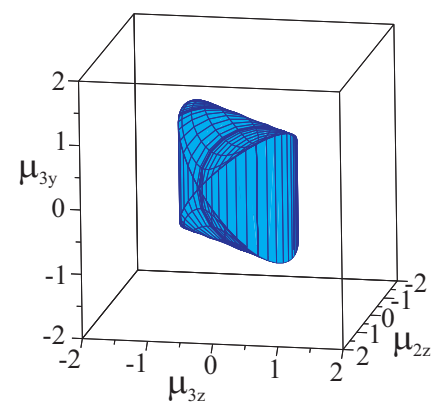

Fig. 5. Joint space of the 3-PPPS robot

In the present case we first make the following change of variables in order to reduce the joint space to a 3 -dimensional subspace: 


$$
\begin{aligned}
& \mu_{1 x}=\rho_{1 x}-\rho_{2 y} \quad \mu_{2 x}=\rho_{2 x}-\rho_{1 y} \quad \mu_{3 x}=\rho_{3 x}+\rho_{2 y} \\
& \mu_{1 y}=\rho_{1 y}-\rho_{1 y}=0 \mu_{2 y}=\rho_{2 y}-\rho_{2 y}=0 \mu_{3 y}=\rho_{3 y}-\rho_{2 y} \\
& \mu_{1 z}=\rho_{1 z}-\rho_{1 z}=0 \mu_{2 z}=\rho_{2 z}-\rho_{1 z} \quad \mu_{3 z}=\rho_{3 z}-\rho_{1 z}
\end{aligned}
$$

Then, the discriminant variety of the kinematic system w.r.t. the projection onto the joint space is given by the union of the following equations

$$
\begin{aligned}
& \mu_{2 z}-\mu_{3 z}=1 \quad 4\left(\mu_{2 z}^{2}-\mu_{2 z} \mu_{3 z}+\mu_{3 z}^{2}\right)=3 \\
& \mu_{2 z}-\mu_{3 z}=-1\left(\mu_{2 z}-\mu_{3 z}\right)^{2}+\mu_{3 y}^{2}=1
\end{aligned}
$$

\begin{tabular}{|c|c|c|}
\hline$\mu_{2 z}$ & $\mu_{3 z}$ & $\mu_{3 y}$ \\
\hline$\left[\mathcal{P}_{1_{R_{2} Z}}, 1, \mu_{2 z}, \mathcal{P}_{2_{R_{2 Z}}}, 1\right]$ & {$\left[\mathcal{P}_{3_{R_{3 Z}}}, 1, \mu_{3 z}, \mathcal{P}_{3_{R_{3 Z}}}, 2\right]$} & {$\left[\mathcal{P}_{1_{R_{3 Y}}}, 1, \mu_{3 y}, \mathcal{P}_{1_{R_{3 Y}}}, 2\right]$} \\
\hline$\left[\mathcal{P}_{2_{R_{2 Z}}}, 1, \mu_{2 z}, \mathcal{P}_{3_{R_{2 Z}}}, 1\right]$ & {$\left[\mathcal{P}_{3_{R_{3 Z}}}, 1, \mu_{3 z}, \mathcal{P}_{3_{R_{3 Z}}}, 2\right]$} & {$\left[\mathcal{P}_{1_{R_{3 Y}}}, 1, \mu_{3 y}, \mathcal{P}_{1_{R_{3 Y}}}, 2\right]$} \\
\hline$\left[\mathcal{P}_{3_{R_{2} Z}}, 1, \mu_{2 z}, \mathcal{P}_{4_{R_{2}}}, 1\right]$ & {$\left[\mathcal{P}_{3_{R_{3}}}, 1, \mu_{3 z}, \mathcal{P}_{3_{R_{3} Z}}, 2\right]$} & {$\left[\mathcal{P}_{1_{R_{3 Y}}}, 1, \mu_{3 y}, \mathcal{P}_{1_{R_{3 Y}}}, 2\right]$} \\
\hline
\end{tabular}

The cell description of the complement of the discriminant variety from the next table comes from [13]. For one variable, $[\mathcal{P}, n, \mu, \mathcal{Q}, m]$ means that the minimum value of $\mu$ is the $n^{t h}$ root of $\mathcal{P}$ and the maximum value is $m^{\text {th }}$ root of $\mathcal{Q}$.

where

$$
\begin{aligned}
& \mathcal{P}_{1_{R_{2 Z}}}: \mu_{2 z}+1=0 \\
& \mathcal{P}_{2_{R_{2 Z}}}: 2 \mu_{2 z}+1=0 \\
& \mathcal{P}_{3_{R_{2 Z}}}: 2 \mu_{2 z}-1=0 \\
& \mathcal{P}_{4_{R_{2 Z}}}: \mu_{2 z}-1=0 \\
& \mathcal{P}_{1_{R_{3 Z}}}: \mu_{2 z}-\mu_{3 z}-1=0 \\
& \mathcal{P}_{2_{R_{3 Z}}}: \mu_{2 z}-\mu_{3 z}+1=0 \\
& \mathcal{P}_{3_{R_{3 Z}}}: 4\left(\mu_{2 z}^{2}-\mu_{2 z} \mu_{3 z}+\mu_{3 z}^{2}\right)-3=0 \\
& \mathcal{P}_{1_{R_{3 Y}}}:\left(\mu_{2 z}-\mu_{3 z}\right)^{2}+\mu_{3 y}^{2}-1=0
\end{aligned}
$$

Taking one point in each of the cells and solving the related zero-dimensional system, it turns out that for the present example, the DKP always admits 16 real roots which corresponds to eight assembly modes for the robot. This result is valid if there is no limit on the passive joints.

\section{Workspace analysis}

Analyzing the workspace consists in computing a partition of this space in regions where the IKP has a fixed number of solutions. As for the joint space analysis, we are only interested in regions of maximal dimension: we can compute the discriminant variety of the kinematics variety w.r.t. the projection onto the position's space and then describe the complement of this variety using an openCAD.

The aim of the analysis is to determine the maximum regions without any singularities, i.e. the aspects of the robot. In these regions, the robot can perform any continuous trajectories. As the Jacobian w.r.t. the joints variables of 
the kinematic system can be factorized into two components, the discriminant variety splits the orientation space into four regions by using the sign of two components.

- Let PP be the regions where $q_{2}^{2}+q_{3}^{2}-1 / 2>0$ and $q_{2}^{2}+q_{4}^{2}-1 / 2>0$.

- Let NN be the regions where $q_{2}^{2}+q_{3}^{2}-1 / 2<0$ and $q_{2}^{2}+q_{4}^{2}-1 / 2<0$.

- Let PN be the regions where $q_{2}^{2}+q_{3}^{2}-1 / 2>0$ and $q_{2}^{2}+q_{4}^{2}-1 / 2<0$.

- Let NP be the regions where $q_{2}^{2}+q_{3}^{2}-1 / 2<0$ and $q_{2}^{2}+q_{4}^{2}-1 / 2>0$.

Each region can be defined by a set of cells.
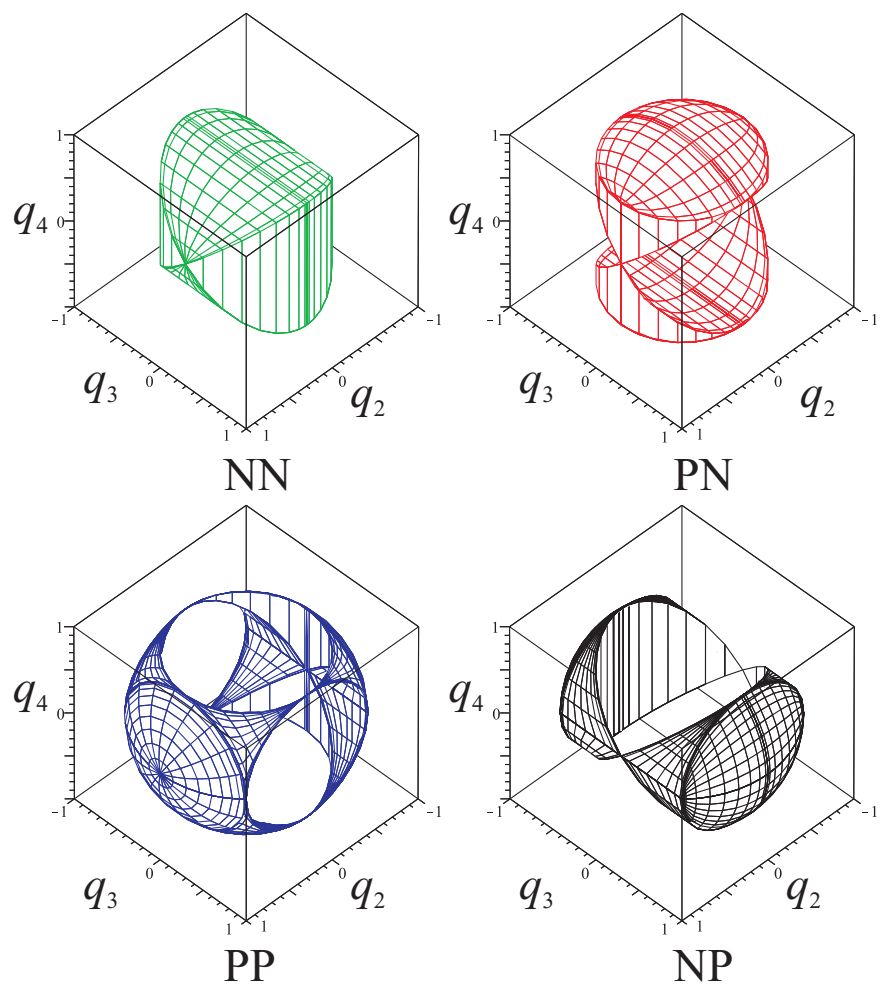

Fig. 6. Workspace of the 3-PPPS robot

\subsection{Cuspidal robots}

The cuspidal character of a mecanism has a different meanings depending on his structure. For a serial mechanism, it is the capability to pass from one solution of the inverse kinematic problem to another one without crossing a singularity in the joint space (the jacobian of the kinematic system w.r.t. the joint variables 
does not cancel) while for a parallel mechanism it is the capability to pass from a solution of the direct kinematic problem to another one without crossing a singularity in the workspace (the jacobian of the kinematic system w.r.t. the position variables does not cancel).

The term "cuspidal" is due to the fact that this capability is linked to the existence of a triple solution to the inverse kinematic problem in the case of a serial mechanism and to the existence of a triple solution to the direct kinematic problem in the case of a parallel mechanism.

For illustrating the way such a property is studied using the SIROPA library, we chose the following 3-PPPS Robot, which is a simplified kinematic version of the manipulator proposed in [5].

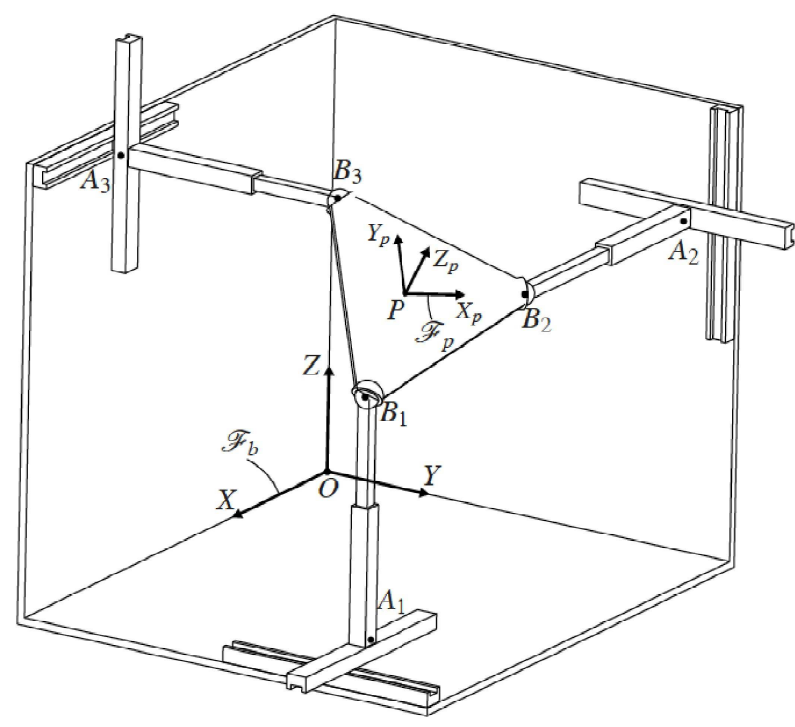

Fig. 7. A 3-PPPS robot with 3 orthogonal prismatic joints.

Let $B_{1}, B_{2}$ and $B_{3}$ be the corners of the moving platform (MP) of side length $r$. Let $\mathcal{F}_{p}\left(P, X_{p}, Y_{p}, Z_{p}\right)$ be the frame attached to the moving platform, its origin $P$ being the centroid of the MP. $Y_{p}$ is parallel to line $\left(B_{1} B_{3}\right)$ and $Z_{p}$ is normal to the MP. Accordingly,

$$
\boldsymbol{b}_{1 p}=[-r \sqrt{3} / 6,-r / 2,0], \boldsymbol{b}_{2 p}=[2 r \sqrt{3} / 6,0,0], \boldsymbol{b}_{3 p}=[-r \sqrt{3} / 6, r / 2,0]
$$

are the Cartesian coordinate vectors of points $B_{1}, B_{2}$ and $B_{3}$ expressed in $\mathcal{F}_{p}$. Likewise, let $\mathcal{F}_{b}(O, X, Y, Z)$ be the frame attached to the base and

$$
\boldsymbol{a}_{1 b}=\left[x_{1}, y_{1}, 0\right], \quad \boldsymbol{a}_{2 b}=\left[0, y_{2}, z_{2}\right], \quad \boldsymbol{a}_{3 b}=\left[x_{3}, 0, z_{3}\right]
$$


be the Cartesian coordinate vectors of points $A_{1}, A_{2}$ and $A_{3}$ expressed in $\mathcal{F}_{b}$.

Let $\boldsymbol{p}=\left[p_{x}, p_{y}, p_{z}\right]^{T}$ be the Cartesian coordinate vector of point $P$, the centroid of the MP, expressed in $\mathcal{F}_{b}$ and let $r$ be equal to 1 . The orientation space of the moving platform is fully represented with the variables $(\phi, \theta, \sigma)$, namely, the azimuth, tilt and torsion angles defined in [2]. The rotation matrix ${ }^{b} \boldsymbol{Q}_{p}$ from $\mathcal{F}_{b}$ to $\mathcal{F}_{p}$ is expressed as follows:

$$
{ }^{b} \boldsymbol{Q}_{p}=\left[\begin{array}{ccc}
C_{\phi} C_{\psi}-S_{\phi} C_{\theta} S_{\psi}-C_{\phi} S_{\psi}-S_{\phi} C_{\theta} C_{\psi} & S_{\phi} S_{\theta} \\
S_{\phi} C_{\psi}+C_{\phi} C_{\theta} S_{\psi}-S_{\phi} S_{\psi}+C_{\phi} C_{\theta} C_{\psi} & -C_{\phi} S_{\theta} \\
S_{\theta} S_{\psi} & S_{\theta} C_{\psi} & C_{\theta}
\end{array}\right]
$$

$C$ and $S$ denoting the cosine and sine functions, respectively. Note that $\phi \in$ $[-\pi, \pi], \theta \in[0, \pi]$ and $\sigma \in[-\pi, \pi]$.

As a consequence, the following constraint equations characterize the geometric model of the 3 - PPPS-manipulator and are obtained by considering the projection of the coordinates of points $B_{i}$ in the plane motion of the two actuated prismatic joints of the $i$ th leg, $i=1, \ldots, 3$ :

$$
\begin{array}{r}
p_{x}-x_{1}=0 \\
p_{y}-y_{1}=0 \\
3 p_{y}-3 y_{2}-2 \sqrt{3} C_{\theta} S_{\sigma}+2 \sqrt{3} C_{\phi}^{2} C_{\theta} S_{\sigma}+2 \sqrt{3} C_{\phi} C_{\sigma} S_{\phi} \\
-\sqrt{6} S_{\theta} C_{\sigma} C_{\phi}-\sqrt{6} S_{\theta} S_{\sigma} S_{\phi}-2 \sqrt{3} S_{\phi} C_{\theta} C_{\sigma} C_{\phi}-2 \sqrt{3} C_{\phi}^{2} S_{\sigma}=0 \\
3 p_{z}-3 z_{2}+\sqrt{3} C_{\theta} S_{\sigma}-\sqrt{3} C_{\phi}^{2} C_{\theta} S_{\sigma}-\sqrt{3} C_{\phi} C_{\sigma} S_{\phi} \\
-\sqrt{6} S_{\theta} C_{\sigma} C_{\phi}-\sqrt{6} S_{\theta} S_{\sigma} S_{\phi}+\sqrt{3} S_{\phi} C_{\theta} C_{\sigma} C_{\phi}-3 C_{\phi} S_{\sigma} S_{\phi} \\
+3 C_{\phi}^{2} C_{\theta} C_{\sigma}+3 C_{\sigma}-3 C_{\phi}^{2} C_{\sigma}+\sqrt{3} C_{\phi}^{2} S_{\sigma}+3 S_{\phi} C_{\theta} S_{\sigma} C_{\phi}=0 \\
6 p_{x}-6 x_{3}+\sqrt{3} C_{\theta} S_{\sigma}+2 \sqrt{3} C_{\phi}^{2} C_{\theta} S_{\sigma}+2 \sqrt{3} C_{\phi} C_{\sigma} S_{\phi}-\sqrt{6} S_{\theta} C_{\sigma} C_{\phi} \\
-\sqrt{6} S_{\theta} S_{\sigma} S_{\phi}+3 \sqrt{2} S_{\theta} S_{\sigma} C_{\phi}-3 \sqrt{2} S_{\theta} C_{\sigma} S_{\phi}-2 \sqrt{3} S_{\phi} C_{\theta} C_{\sigma} C_{\phi} \\
+3 C_{\theta} C_{\sigma}-6 C_{\phi}^{2} C_{\theta} C_{\sigma}+6 C_{\phi} S_{\sigma} S_{\phi}-3 C_{\sigma}+6 C_{\phi}^{2} C_{\sigma}-2 \sqrt{3} C_{\phi}^{2} S_{\sigma} \\
-6 S_{\phi} C_{\theta} S_{\sigma} C_{\phi}+3 \sqrt{3} S_{\sigma}=0 \\
6 p_{z}-6 z_{3}+\sqrt{3} C_{\theta} S_{\sigma}-4 \sqrt{3} C_{\phi}^{2} C_{\theta} S_{\sigma}-4 \sqrt{3} C_{\phi} C_{\sigma} S_{\phi} \\
-\sqrt{6} S_{\theta} C_{\sigma} C_{\phi}-\sqrt{6} S_{\theta} S_{\sigma} S_{\phi}+3 \sqrt{2} S_{\theta} S_{\sigma} C_{\phi}-3 \sqrt{2} S_{\theta} C_{\sigma} S_{\phi} \\
+4 \sqrt{3} S_{\phi} C_{\theta} C_{\sigma} C_{\phi}+3 C_{\theta} C_{\sigma}+3 C_{\sigma}+4 \sqrt{3} C_{\phi}^{2} S_{\sigma}-3 \sqrt{3} S_{\sigma}=0
\end{array}
$$

It is noteworthy that the translational and rotational motions of the moving platform of the 3 - PPPS-manipulator shown in Fig. 4.1 can be decoupled. In order to highlight this decoupling, the following change of variables is made:

$$
\begin{array}{ccc}
X_{1}=\frac{x_{1}+x_{3}}{2} & Y_{1}=y_{2}-y_{1} & Z_{2}=z_{2}-z_{3} \\
X_{3}=x_{3}-x_{1} & Y_{2}=\frac{y_{1}+y_{2}}{2} & Z_{3}=\frac{z_{2}+z_{3}}{2}
\end{array}
$$

It is apparent that the translational motions of the MP depend only on variables $X_{1}, Y_{2}$ and $Z_{3}$, whereas its rotational motions depend only on variables 
$X_{3}, Y_{1}$ and $Z_{2}$. Due to the decoupling of the translational and rotational motions, one can, for exemple compute (and draw) the singularity surface in the joint space defined by the translational variables.

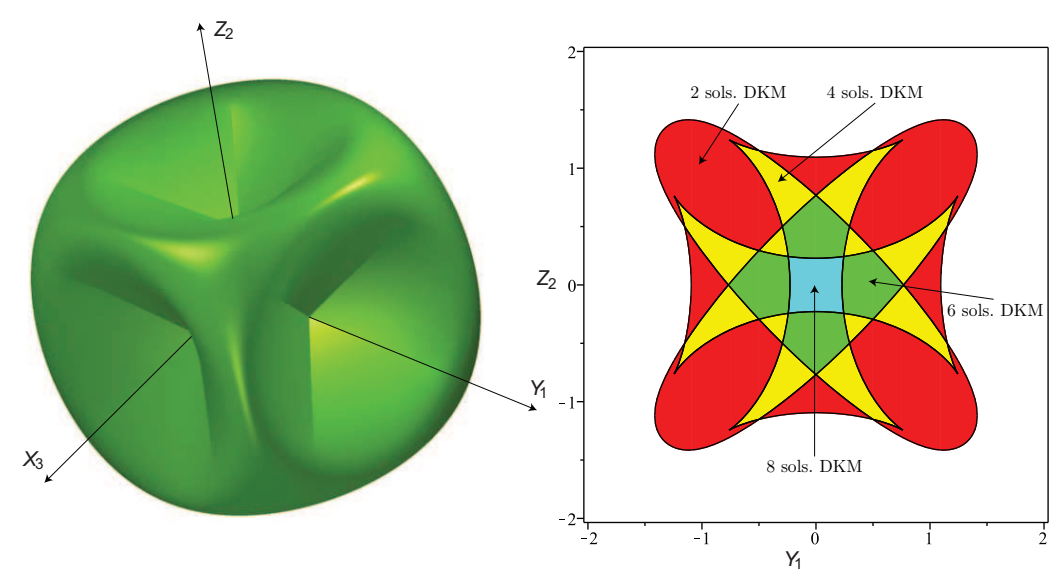

Fig. 8. Singularity surface and CAD-partition of the joint space

In the present case, the projection of this singularity surface onto the translational variables is equal to the discriminant variety of the kinematics equations w.r.t. the projection onto the the translational variables and thus defines a partition of this space in region where the direct kinematic problem has a constant number of solutions.

\section{References}

[1] Dennis S. Arnon, George E. Collins, and Scott McCallum. Cylindrical algebraic decomposition i: the basic algorithm. SIAM J. Comput., 13(4):865-877, nov 1984.

[2] Ilian A Bonev and Jeha Ryu. Orientation workspace analysis of 6-dof parallel manipulators. In Proceedings of the ASME. 1999.

[3] Damien Chablat and Philippe Wenger. Working modes and aspects in fully parallel manipulators. In Proceedings. 1998 IEEE International Conference on Robotics and Automation (Cat. No. 98CH36146), volume 3, pages 1964-1969. IEEE, 1998.

[4] Chao Chen, Thibault Gayral, Stéphane Caro, Damien Chablat, Guillaume Moroz, and Sajeeva Abeywardena. A six degree of freedom epicyclic-parallel manipulator. Journal of Mechanisms and Robotics, 4(4):41011, 2012.

[5] Chao Chen, William John Heyne, and Daniel Jackson. A new 6-dof 3-legged parallel mechanism for force-feedback interface. In Proceedings of 2010 IEEE/ASME International Conference on Mechatronic and Embedded Systems and Applications, pages 539-544. IEEE, 2010.

[6] Solen Corvez and Fabrice Rouillier. Using computer algebra tools to classify serial manipulators. In International Workshop on Automated Deduction in Geometry, pages 31-43. Springer, 2002. 
[7] Manfred L Husty. An algorithm for solving the direct kinematics of general stewartgough platforms. Mechanism and Machine Theory, 31(4):365-379, 1996.

[8] Ranjan Jha, Damien Chablat, Luc Baron, Fabrice Rouillier, and Guillaume Moroz. Workspace, Joint space and Singularities of a family of Delta-Like Robot. Mechanism and Machine Theory, 127:73-95, Sep 2018.

[9] Wisama Khalil and Etienne Dombre. Modeling, identification and control of robots. Butterworth-Heinemann, 2004.

[10] Daniel Lazard and Fabrice Rouillier. Solving parametric polynomial systems. Journal of Symbolic Computation, 42(6):636-667, 2007.

[11] Montserrat Manubens, Guillaume Moroz, Damien Chablat, Philippe Wenger, and Fabrice Rouillier. Cusp points in the parameter space of degenerate 3-rpr planar parallel manipulators. Journal of mechanisms and robotics, 4(4):41003, 2012.

[12] Jean-Pierre Merlet. Parallel robots, volume 128. Springer Science \& Business Media, 2006.

[13] Guillaume Moroz, Fabrice Rouiller, Damien Chablat, and Philippe Wenger. On the determination of cusp points of 3-rpr parallel manipulators. Mechanism and Machine Theory, 45(11):1555-1567, 2010.

[14] Philippe Wenger and Damien Chablat. Kinetostatic analysis and solution classification of a class of planar tensegrity mechanisms. Robotica, 37(7):1214-1224, 2019. 